\title{
A Characterization of Central BMO Space via the Commutator of Fractional Hardy Operator
}

\author{
Lei Zhang $\mathbb{D}$ and Shaoguang Shi \\ Department of Mathematics, Linyi University, Linyi 276005, China \\ Correspondence should be addressed to Lei Zhang; zhanglei-0335@163.com
}

Received 25 March 2020; Accepted 23 April 2020; Published 31 May 2020

Guest Editor: Lishan Liu

Copyright (c) 2020 Lei Zhang and Shaoguang Shi. This is an open access article distributed under the Creative Commons Attribution License, which permits unrestricted use, distribution, and reproduction in any medium, provided the original work is properly cited.

This paper is devoted in characterizing the central BMO $\left(\mathbb{R}^{n}\right)$ space via the commutator of the fractional Hardy operator with rough kernel. Precisely, by a more explicit decomposition of the operator and the kernel function, we will show that if the symbol function belongs to the central BMO $\left(\mathbb{R}^{n}\right)$ space, then the commutator are bounded on Lebesgue space. Conversely, the boundedness of the commutator implies that the symbol function belongs to the central BMO $\left(\mathbb{R}^{n}\right)$ space by exploiting the center symmetry of the Hardy operator deeply.

\section{Introduction}

In this paper, we focus on the need for characterizing the central BMO $\left(\mathbb{R}^{n}\right)$ space via the boundedness of the commutators of the following fractional Hardy operators

$$
\begin{aligned}
H_{\Omega, \alpha} f(x) & =\frac{1}{|x|^{n-\alpha}} \int_{|y|<|x|} \Omega(x-y) f(y) d y, \\
H_{\Omega, \alpha}^{*} f(x) & =\int_{|y| \geq|x|} \frac{\Omega(x-y) f(y)}{|y|^{n-\alpha}} d y, \\
0 & <\alpha<n .
\end{aligned}
$$

$H_{\Omega, \alpha}^{*}$ is the dual operator of $H_{\Omega, \alpha}$. Here, $\Omega$ satisfies

$$
\begin{gathered}
\Omega(t x)=\Omega(x), \forall t>0, x \in \mathbb{R}^{n} ; \\
\int_{\mathbb{S}^{n-1}} \Omega\left(x^{\prime}\right) d \sigma\left(x^{\prime}\right)=0 ; \\
\Omega \in L^{q}\left(\mathbb{S}^{n-1}\right) \forall q>1 ;
\end{gathered}
$$

and $\mathbb{S}^{n-1}=\left\{x \in \mathbb{R}^{n}:|x|=1\right\}$ denotes the unit sphere in $\mathbb{R}^{n}$.

For a function $b$, the commutators of $H_{\Omega, \alpha}$ and $H_{\Omega, \alpha}^{*}$ can be written as

$$
\begin{aligned}
{\left[b, H_{\Omega, \alpha}\right] f:=b\left(H_{\Omega, \alpha} f\right)-H_{\Omega, \alpha}(b f), } \\
{\left[b, H_{\Omega, \alpha}^{*}\right] f:=b\left(H_{\Omega, \alpha}^{*} f\right)-H_{\Omega, \alpha}^{*}(b f) . }
\end{aligned}
$$

In [1], Fu et al. considered the boundedness of $H_{\Omega, \alpha}$ and $\left[b, H_{\Omega, \alpha}\right]$ on homogeneous Herz spaces and Lebesgue spaces under the assumption that $\Omega$ satisfies (2) and (4). We recall the results from ([1], Proposition 3.1 and Theorem 3.1) as

Suppose that

$$
\left\{\begin{array}{l}
1<p_{1}, p_{2}<\infty \text { with } \frac{1}{p_{1}}=\frac{1}{p_{2}}+\frac{\alpha}{n} ; \\
\frac{1}{p_{1}}+\frac{1}{s}+\frac{1}{q}=1 \text { for some } s>p_{1}^{\prime}=\frac{p_{1}}{p_{1}-1} \\
\Omega \text { satisfies }(1.1) \text { and }(1.3) ; \\
b \in \mathrm{CBMO}^{\max \left\{p_{2}, s\right\}}\left(\mathbb{R}^{n}\right) .
\end{array}\right.
$$


Then,

$$
\left\{\begin{array}{l}
H_{\Omega, \alpha} \text { is bounded from } L^{p_{1}}\left(\mathbb{R}^{n}\right) \text { to } L^{p_{2}}\left(\mathbb{R}^{n}\right) ; \\
{\left[b ; H_{\Omega, \alpha}\right] \text { is bounded from } L^{p_{1}}\left(\mathbb{R}^{n}\right) \text { to } L^{p_{2}}\left(\mathbb{R}^{n}\right) .}
\end{array}\right.
$$

For the boundedness of the classical fractional Hardy operator, see [2]. For a ball $B_{r}:=B(0, r) \subset \mathbb{R}^{n}$ (i.e., a ball centered at 0 with radius $r>0)$ and $p \geq 1, \operatorname{CBMO}^{p}\left(\mathbb{R}^{n}\right)$ is the central BMO $\left(\mathbb{R}^{n}\right)$ function space, which was introduced by $\mathrm{Lu}$ and Yang [3] via the norm

$$
\begin{aligned}
\|b\|_{\mathrm{CBMO}^{p}\left(\mathbb{R}^{n}\right)} & =\sup _{r>0}\left(\frac{1}{\left|B_{r}\right|} \int_{B_{r}}\left|b(x)-b_{B_{r}}\right|^{p} d x\right)^{1 / p} \text { with } b_{B_{r}} \\
& =\frac{1}{\left|B_{r}\right|} \int_{B_{r}} b(x) d x .
\end{aligned}
$$

It is easy to see that CBMO $\left(\mathbb{R}^{n}\right)$ can be understood as a local version of the classical BMO $\left(\mathbb{R}^{n}\right)$ space at the origin [4] and

$$
\begin{aligned}
\operatorname{BMO}\left(\mathbb{R}^{n}\right) & \subset \operatorname{CBMO}\left(\mathbb{R}^{n}\right), \\
\operatorname{CBMO}^{p>1}\left(\mathbb{R}^{n}\right) & \subset \operatorname{CBMO}\left(\mathbb{R}^{n}\right) .
\end{aligned}
$$

Hence, the famous John-Nirenberg inequality for BMO $\left(\mathbb{R}^{n}\right)$ space is not true for CBMO $\left(\mathbb{R}^{n}\right)$ space, which reveals that they have quite different properties.

In the study of harmonic analysis, the characterization of function spaces via the boundedness of the commutators plays an important role in the field of PDEs, see, for example, [5-11] and the references therein. However, there are less attention paid for the commutators with rough kernels since the characterization depends heavily on the smoothness of the kernel function $\Omega$. Under the premise that $\Omega$ is smooth enough, i.e., $\Omega \in C^{\infty}\left(\mathbb{S}^{n-1}\right)$ or $\Omega \in \operatorname{Lip}_{1}\left(\mathbb{S}^{n-1}\right)$, see, for example, [12-15]. It is difficult to weaken the smoothness of $\Omega$, Chen and Ding [16] considered a characterization of BMO
$\left(\mathbb{R}^{n}\right)$ space under the condition that $\Omega$ satisfies the following Hölder condition of log type

$$
\begin{aligned}
\left|\Omega\left(x^{\prime}\right)-\Omega\left(y^{\prime}\right)\right| & \leq \frac{A}{\left(\log \left(2 /\left|x^{\prime}-y^{\prime}\right|\right)\right)^{\gamma}} \quad \text { with } A \\
& >0, \gamma>1, x^{\prime}, y^{\prime} \in \mathbb{S}^{n-1} .
\end{aligned}
$$

It is easy to check that (10) is weaker than the Lipschitz condition and stronger than the condition (4). For $q \geq 1$ and $\Omega \in L^{q}\left(\mathbb{S}^{n-1}\right)$, we call $\Omega$ satisfies the $L^{q}$-Dini condition if $\int_{0}^{1}\left(\left(w_{q}(\delta)\right) /(\delta)\right)<\infty$, where $w_{q}(\delta)$ is defined as

$$
\begin{aligned}
w_{q}(\delta)= & \sup _{\|\tau\| \leq \delta}\left(\int_{\mathbb{S}^{n-1}}\left|\Omega\left(\tau x^{\prime}\right)-\Omega\left(x^{\prime}\right)\right|^{q} d \sigma\left(x^{\prime}\right)\right)^{1 / q} \\
& \text { with }\|\tau\|=\sup _{x^{\prime} \in \mathbb{S}^{n-1}}\left|\tau x^{\prime}-x^{\prime}\right| .
\end{aligned}
$$

As a useful supplement of [1], we give a characterization of the CBMO $\left(\mathbb{R}^{n}\right)$ space via the boundedness of $\left[b, H_{\Omega, \alpha}\right]$ and $\left[b, H_{\Omega, \alpha}^{*}\right]$ as follows.

Theorem 1. Let $1<p_{1}, p_{2}<\infty$ with $\left(1 / p_{1}\right)=\left(1 / p_{2}\right)+(\alpha / n)$ and $\left(1 / p_{1}\right)+(1 / s)(1 / q)+1$ for somes $>p_{1}{ }^{\prime}$.

(a) If

$$
\left\{\begin{array}{l}
\Omega \text { satisfies }(1.1) \text { and }(1.3) ; \\
b \in C B M O^{\max }\left\{p_{2}, s\right\}\left(\mathbb{R}^{n}\right)
\end{array}\right.
$$

then

$\left\{\begin{array}{l}{\left[b, H_{\Omega, \alpha}\right] \text { is bounded from } L^{p_{1}}\left(\mathbb{R}^{n}\right) \text { to } L^{p_{2}}\left(\mathbb{R}^{n}\right) ;} \\ {\left[b, H_{\Omega, \alpha}\right] \text { is bounded from } L^{p_{1}}\left(\mathbb{R}^{n}\right) \text { to } L^{p_{2}}\left(\mathbb{R}^{n}\right) .}\end{array}\right.$

(b) if

$$
\left\{\begin{array}{l}
\Omega \text { satisfies }(1.1),(1.2) \text {, and }(1.4) ; \\
{\left[b, H_{\Omega, \alpha}\right] \text { is bounded from } L^{p_{1}}\left(\mathbb{R}^{n}\right) \text { to } L^{p_{2}}\left(\mathbb{R}^{n}\right) ; \text { then } b \in C B M O\left(\mathbb{R}^{n}\right) .} \\
{\left[b, H_{\Omega, \alpha}\right] \text { is bounded from } L^{p_{1}}\left(\mathbb{R}^{n}\right) \text { to } L^{p_{2}}\left(\mathbb{R}^{n}\right)}
\end{array}\right.
$$

A part of Theorem 1 has been proven in [1], we will show the rest of Theorem 1 in Section 2. In what follows, we will denote $C$ by a positive constant which may vary from line to line. The symbol $A \leq B$ means $A \leq C B$ and $\mathbb{Z}$ for the set of all integers. Last, but not least, $B_{r}:=B(0, r), B_{k}:=B_{2^{k}}$, $C_{k}:=B_{k} \backslash B_{k-1}$, and $\chi_{k}:=\chi_{C_{k}}$ with $k \in \mathbb{Z}$.

\section{Proof of Theorem 1.1}

We prove Theorem 1 in this section. To do so, we need one lemma about the estimates of the kernel function $\Omega$, which plays a key role in the proof. 
Lemma 2. Let $\Omega$ satisfy (2) and (10). Then,

(a) $|\Omega(x-y)-\Omega(x)| \leq C /(\log (|x| /|y|))^{\gamma}$ with $\gamma$ be given in (10) and $|x| \geq 4|y|$. (b) If $\Omega$ furthermore satisfies the $L^{q \geq 1}-D i n i$ condition, then, there is a $C>0$ such that for $0<C<1 / 2, r>0$, $x \in \mathbb{R}^{n}$ with $|x|<C r$; we have

$$
\left\{\begin{array}{l}
\left(\int_{r<|y|<2 r}|\Omega(y-x)-\Omega(y)|^{q} d y\right)^{1 / q} \leq C r^{n / q} \int_{|x| / 2 r}^{|x| / r} \frac{w_{q}(\delta)}{\delta} d \delta ; \\
\left(\int_{r<|y|<2 r} \frac{|\Omega(y-x)-\Omega(y)|^{q}}{|y|^{(n-\alpha) q}} d y\right)^{1 / q} \leq C r^{-\left(n / q^{\prime}\right)+\alpha} \int_{|x| / 2 r}^{|x| / r} \frac{w_{q}(\delta)}{\delta} d \delta \quad \text { with } 0<\alpha<\frac{n}{p_{1}} .
\end{array}\right.
$$

Proof. We give the proof by a slight modification from [17]. For $|x| \geq 4|y|$, we first show that

$$
\begin{gathered}
\left|(x-y)^{\prime}-x^{\prime}\right| \leq \frac{3|y|}{|y|}, \\
|1 /| x|-1 /| x-y|| \leq C|y| /|x|^{2} .
\end{gathered}
$$

Indeed, the first inequality can be obtained immediately from ([18], Lemma 2). Since

$$
|1 /| x|-1 /| x-y|| \leq 4|y| / 3|x|^{2} \leq C|y| /|x|^{2},
$$

(a) can be shown by (2) and (10) as

$$
\begin{aligned}
|\Omega(x-y)-\Omega(x)| & \leq \frac{C}{\left(\log \left(2 /\left|(x-y)^{\prime}-x^{\prime}\right|\right)\right)^{\gamma}} \\
& \leq \frac{C}{(\log (|x| /|y|))^{\gamma}} .
\end{aligned}
$$

We are left to show (b). The first estimate can be obtained directly by the $L^{q}-$ Dini condition as

$$
\begin{aligned}
& \left(\int_{r<|y|<2 r}|\Omega(y-x)-\Omega(y)|^{q} d y\right)^{1 / q} \\
& \quad=\left(\int_{r}^{2 r} \int_{\mathbb{S}^{n-1} \mid}\left|\Omega\left(y^{\prime}-t^{-1} x^{\prime}\right)-\Omega\left(y^{\prime}\right)\right|^{q} d \delta\left(y^{\prime}\right) t^{n-1} d t\right)^{1 / q} \\
& \quad \leq C r^{n / q} \int_{|x| / 2 r}^{|x| / r} \frac{w_{q}(\delta)}{\delta} d \delta
\end{aligned}
$$

and the estimate

$$
\int_{\mathbb{S}^{n-1}}\left|\Omega\left(y^{\prime}-t^{-1} x^{\prime}\right)-\Omega\left(y^{\prime}\right)\right|^{q} d \delta\left(y^{\prime}\right) \leq C w_{q}^{q}\left(\frac{|x|}{t}\right) .
$$

follows from ([19], p.65-77).
Accordingly, the second can be deduced similarly. In fact,

$$
\begin{gathered}
\left(\int_{r<|y|<2 r} \frac{|\Omega(y-x)-\Omega(y)|^{q}}{|y|^{(n-\alpha) q}} d y\right)^{1 / q} \\
=C r^{-\left(n / q^{\prime}\right)+\alpha}\left(\int_{r}^{2 r} \int_{\mathbb{S}^{n-1}} \mid \Omega\left(y^{\prime}-t^{-1} x^{\prime}\right)\right. \\
\left.-\left.\Omega\left(y^{\prime}\right)\right|^{q} d \delta\left(y^{\prime}\right) \frac{d t}{t}\right)^{1 / q} \\
\leq \operatorname{Cr}^{-\left(n / q^{\prime}\right)+\alpha} \int_{|x| / 2 r}^{|x| / r} \frac{w_{q}(\delta)}{\delta} d \delta
\end{gathered}
$$

which is the desired one.

The following is the boundedness of the fractional Hardy operators.

Theorem 3. Let $1<p_{1}, p_{2}<\infty$ with $\left(1 / p_{1}\right)=\left(1 / p_{2}\right)+(\alpha / n)$ and $\Omega$ satisfy (2) and (4). Then, both $H_{\Omega, \alpha}$ and $H_{\Omega, \alpha}^{*}$ are bounded from $L^{p_{1}}\left(\mathbb{R}^{n}\right)$ to $L^{p_{2}}\left(\mathbb{R}^{n}\right)$.

Proof. Since the $\left(L^{p_{1}}\left(\mathbb{R}^{n}\right), L^{p_{2}}\left(\mathbb{R}^{n}\right)\right)$ boundedness for $H_{\Omega, \alpha}$ is contained in ([1], Proposition 3.1), it is enough to check the boundedness for $H_{\Omega, \alpha}^{*}$. Namely, the task is now left to show that there exist constants $C>0$ such that for any $f \in L^{p_{1}}\left(\mathbb{R}^{n}\right)$, one has

$$
\left\|H_{\Omega}^{*} f\right\|_{L^{p_{2}}\left(\mathbb{R}^{n}\right)} \leq C\|f\|_{L^{p_{1}}\left(\mathbb{R}^{n}\right)} .
$$

To do so, we first recall a useful estimates from [1] as

$$
\begin{aligned}
|x-y| & \leq|x|+2^{i} \leq 2^{k+1} \& \int_{C_{i}}|\Omega(x-y)|^{q} d y \\
& \leq \int_{0}^{2^{k+1}} \int_{\mathbb{S}^{n-1}}\left|\Omega\left(x^{\prime}\right)\right|^{q} d \delta\left(x^{\prime}\right) t^{n-1} d t \leq C 2^{k n}
\end{aligned}
$$


for $x \in C_{k}, y \in C_{i}$, and $i \leq k$. Since

$$
\begin{aligned}
\int_{\mathbb{R}^{n}}\left|H_{\Omega}^{*} f(x)\right|^{p_{2}} d x \leq & \sum_{k=-\infty}^{+\infty} \int_{C_{k}}\left|\int_{|x| \leq y \mid \leq 2^{k n}} \frac{\Omega(x-y) f(y)}{|y|^{n-\alpha}} d y\right|^{p_{2}} d x \\
& +\sum_{k=-\infty}^{+\infty} \int_{C_{k}}\left|\int_{|y|>2^{k n}} \frac{\Omega(x-y) f(y)}{|y|^{n-\alpha}} d y\right|^{p_{2}} d x \\
:= & I_{1}+I_{2} .
\end{aligned}
$$

Applying Hölder's inequality to $q, p_{1}, s$ for $s>1$ and (24), we have

$$
\begin{aligned}
& I_{1} \leq \sum_{k=-\infty}^{+\infty} 2^{-k(n-\alpha) p_{2}} \int_{C_{k}} \mid \sum_{i=-\infty}^{k}\left(\int_{C_{i}}|\Omega(x-y)|^{q} d y\right)^{1 / q} \\
& \left.\cdot\left(\int_{C_{i}}|f(y)|^{p_{1}} d y\right)^{1 / p_{1}}\left(\int_{C_{i}} d y\right)^{1 / s}\right|^{p_{2}} d x \\
& =C \sum_{k=-\infty}^{+\infty}\left|\sum_{i=-\infty}^{k} 2^{(i-k) n / s}\left(\int_{C_{i}}|f(y)|^{p_{1}} d y\right)^{1 / p_{1}}\right|^{p_{2}} \\
& \leq C \sum_{k=-\infty}^{+\infty} \sum_{i=-\infty}^{k} 2^{(i-k) n p_{2} / 2 s}\|f\|_{L^{p_{1}}\left(B_{i}\right)}^{p_{2}}\left(\sum_{i=-\infty}^{k} 2^{(i-k) n p_{2}^{\prime} / 2 s}\right)^{p_{2} / p_{2}^{\prime}} \\
& \leq C \sum_{k=-\infty}^{+\infty} \sum_{i=-\infty}^{k} 2^{(i-k) n p_{2} / 2 s}\|f\|_{L^{p_{1}}\left(B_{i}\right)}^{p_{2}} \\
& \leq C \sum_{i=-\infty}^{+\infty}\|f\|_{L^{p_{1}}\left(B_{i}\right)}^{p_{2}} \sum_{k=i}^{+\infty} 2^{(i-k) n p_{2} / 2 s} \leq C\|f\|_{L^{p_{1}}\left(\mathbb{R}^{n}\right)}^{p_{2}}, \\
& I_{2} \leq \sum_{k=-\infty}^{+\infty} \int_{B_{k}} \mid \sum_{i=k}^{\infty}\left(\int_{C_{i}}|\Omega(x-y)|^{q} d y\right)^{1 / q} \\
& \left.\cdot\left(\int_{C_{i}}|f(y)|^{p_{1}} d y\right)^{1 / p_{1}}\left(\int_{C_{i}}|y|^{-(n-\alpha) s} d y\right)^{1 / s}\right|^{p_{2}} d x \\
& \leq C \sum_{k=-\infty}^{+\infty}\left|\sum_{i=k}^{+\infty} 2^{(k-i) n / p_{2}}\|f\|_{L^{p_{1}\left(B_{i}\right)}}\right|^{p_{2}} \\
& \leq C \sum_{k=-\infty}^{+\infty} \sum_{i=k}^{+\infty} 2^{(k-i) n / 2 p_{2}}\|f\|_{L^{p_{1}}\left(B_{i}\right)}^{p_{2}} \\
& \leq C \sum_{i=-\infty}^{+\infty}\|f\|_{L^{p_{1}}\left(B_{i}\right)}^{p^{2^{2}}} \sum_{k=-\infty}^{i} 2^{(k-i) n / 2 p_{2}} \leq C\|f\|_{L^{p_{1}}\left(\mathbb{R}^{n}\right)}^{p_{2}},
\end{aligned}
$$

which is our desired result.

Now, we can prove Theorem 1. Without loss of generality, we can assume that $\left.\|b\|_{C B M O^{\max }\left\{p_{2}, s\right\}} \mathbb{R}^{n}\right)=1$ in the proof of (a) since $b \in \mathrm{CBMO}^{\max }\left\{p_{2}, s\right\}\left(\mathbb{R}^{n}\right)$. We see at once that the
$\left(L^{p_{1}}\left(\mathbb{R}^{n}\right), L^{p_{2}}\left(\mathbb{R}^{n}\right)\right)$ boundedness of $\left[b, H_{\Omega, \alpha}\right]$ is just ([1], Theorem 3.1). To complete the proof of (a), what is left is to show is that

$$
\left\|\left[b, H_{\Omega, \alpha}^{*}\right] f\right\|_{L^{p_{2}\left(\mathbb{R}^{n}\right)}} \leq C\|f\|_{L^{p_{1}}\left(\mathbb{R}^{n}\right)} .
$$

It is easy to check that

$$
\begin{aligned}
\int_{\mathbb{R}^{n}} \mid & {\left.\left[b, H_{\Omega, \alpha}^{*}\right] f(x)\right|^{p_{2}} d x } \\
= & \sum_{k=-\infty}^{+\infty} \int_{C_{k}}\left|\int_{|y| \geq|x|} \frac{\Omega(x-y)(b(x)-b(y)) f(y)}{|y|^{n-\alpha}} d y\right|^{p_{2}} d x \\
= & \sum_{k=-\infty}^{+\infty} \int_{C_{k}} \mid \int_{2^{k n} \geq|y| \geq|x|} \frac{\Omega(x-y)(b(x)-b(y)) f(y)}{|y|^{n-\alpha}} d y \\
& +\left.\int_{|y|>2^{k n}} \frac{\Omega(x-y)(b(x)-b(y)) f(y)}{|y|^{n-\alpha}} d y\right|^{p_{2}} d x \\
\leq & C \sum_{k=-\infty}^{+\infty} \int_{C_{k}}\left|\int_{|x| \leq|y| \leq 2^{k^{k n}}} \frac{\Omega(x-y)(b(x)-b(y)) f(y)}{|y|^{n-\alpha}} d y\right|^{p_{2}} d x \\
& +C \sum_{k=-\infty}^{+\infty} \int_{C_{k} \mid}\left|\int_{|y|>2^{k n}} \frac{\Omega(x-y)(b(x)-b(y)) f(y)}{|y|^{n-\alpha}} d y\right|^{p_{2}} d x \\
:= & J_{1}+J_{2} .
\end{aligned}
$$

Using Hölder's inequality, we have

$$
\begin{aligned}
J_{1} \leq C & \sum_{k=-\infty}^{+\infty} \int_{C_{k}}\left|\frac{1}{|x|^{n-\alpha}} \int_{|y| \leq 2^{k n}} \Omega(x-y)(b(x)-b(y)) f(y) d y\right|^{p_{2}} d x \\
\leq C & \sum_{k=-\infty}^{+\infty} 2^{-k(n-\alpha) p_{2}} \int_{B_{k}} \mid \sum_{i=-\infty}^{k} \int_{B_{i}} \Omega(x-y)(b(x) \\
& -b(y))\left.f(y) d y\right|^{p_{2}} d x \leq C\|f\|_{L^{p_{1}}\left(\mathbb{R}^{n}\right)}^{p_{2}} .
\end{aligned}
$$

For the term $J_{2}$, we see at once that

$$
\begin{aligned}
J_{2} \leq & C \sum_{k=-\infty}^{+\infty} \int_{C_{k}}\left|\sum_{i=k}^{+\infty} \int_{C_{i}} \frac{\Omega(x-y)\left(b(x)-b_{B_{k}}\right) f(y)}{|y|^{n-\alpha}} d y\right|^{p_{2}} d x \\
& +C \sum_{k=-\infty}^{+\infty} \int_{C_{k}}\left|\sum_{i=k}^{+\infty} \int_{C_{i}} \frac{\Omega(x-y)\left(b(y)-b_{B_{k}}\right) f(y)}{|y|^{n-\alpha}} d y\right|^{p_{2}} d x \\
:=J_{21}+J_{22} . &
\end{aligned}
$$


The Hölder inequality, along with (24), implies

$$
\begin{aligned}
& J_{21} \leq C \sum_{k=-\infty}^{+\infty} \int_{C_{k}}\left|b(x)-b_{B_{k}}\right|^{p_{2}}\left|\sum_{i=k}^{+\infty} \int_{C_{i}} \frac{\Omega(x-y) f(y)}{|y|^{n-\alpha}} d y\right|^{p_{2}} d x \\
& \leq C \sum_{k=-\infty}^{+\infty} \int_{B_{k}}\left|b(x)-b_{B_{k}}\right|^{p_{2}} \mid \sum_{i=k}^{+\infty}\|f\|_{L^{p_{1}}\left(B_{i}\right)} \\
& \left.\cdot\left(\int_{C_{i}}|\Omega(x-y)|^{q} d y\right)^{1 / q}\left(\int_{C_{i}}|y|^{-(n-\alpha) s} d y\right)^{1 / s}\right|^{p_{2}} d x \\
& \leq C \sum_{k=-\infty}^{+\infty} \sum_{i=k}^{+\infty} 2^{(k-i) n / 2}\|f\|_{L^{p_{1}}\left(B_{i}\right)}^{p_{2}}\left(\sum_{i=k}^{+\infty} 2^{(k-i) n p_{2}^{\prime} / 2 p_{2}}\right)^{p_{2} / p_{2}^{\prime}} \\
& \leq C\|f\|_{L^{p}\left(\mathbb{R}^{n}\right)}^{p} \text {. }
\end{aligned}
$$

The term $J_{22}$ need a further decomposition as follows:

$$
\begin{aligned}
J_{22} \leq & C \sum_{k=-\infty}^{+\infty} \int_{B_{k}}\left|\sum_{i=k}^{+\infty} \int_{C_{i}} \frac{\Omega(x-y)\left(b(y)-b_{B_{i}}\right) f(y)}{|y|^{n-\alpha}} d y\right|^{p_{2}} d x \\
& +C \sum_{k=-\infty}^{+\infty} \int_{B_{k}}\left|\sum_{i=k}^{+\infty} \int_{C_{i}} \frac{\Omega(x-y)\left(b_{B_{i}}-b_{B_{k}}\right) f(y)}{|y|^{n-\alpha}} d y\right|^{p_{2}} d x \\
:= & J_{221}+J_{222} .
\end{aligned}
$$

Applying the Hölder inequality, we deduce

$$
\begin{aligned}
J_{221} \leq & C \sum_{k=-\infty}^{+\infty} \int_{B_{k}} \mid \sum_{i=k}^{+\infty}\left(\int_{C_{i}} \frac{|f(y)|^{p_{1}}}{\mid y-\alpha) p_{1}} d y\right)^{1 / p} \\
& \left.\cdot\left(\int_{C_{i}}|\Omega(x-y)|^{q} d y\right)^{1 / q}\left(\int_{C_{i}}\left|\left(b(y)-b_{B_{i}}\right)\right|^{s} d y\right)^{1 / s}\right|^{p_{2}} d x \\
\leq & C\|b\|_{\text {CBMO }}^{p}\left(\mathbb{R}^{n}\right) \sum_{k=-\infty}^{+\infty}\left|\sum_{i=k}^{+\infty} 2^{(k-i) n / p}\|f\|_{L^{p}\left(B_{i}\right)}\right|^{p} \\
\leq & C \sum_{k=-\infty}^{+\infty} \sum_{i=k}^{+\infty} 2^{(k-i) n / 2}\|f\|_{L^{p_{1}}\left(B_{i}\right)}^{p_{2}}\left(\sum_{i=k}^{+\infty} 2^{(k-i) n p_{2}^{\prime} / 2 p_{2}}\right)^{p_{2} / p_{2}^{\prime}} \\
\leq & C\|f\|_{L^{p}\left(\mathbb{R}^{n}\right)}^{p} .
\end{aligned}
$$

From the fact that

$$
\begin{aligned}
\left|b_{B_{k}}-b_{B_{i}}\right| & \leq \sum_{j=k}^{i-1}\left|b_{B_{j}}-b_{B_{j+1}}\right| \\
& \leq \sum_{j=k}^{i-1} \frac{1}{\left|B_{j}\right|} \int_{B_{j+1}}\left|b(y)-b_{B_{j+1}}\right| d y \\
& \leq C(i-k)\|b\|_{\mathrm{CBMO}^{p_{2}}\left(B_{i}\right)},
\end{aligned}
$$

the term $J_{222}$ can be estimated as follows:

$$
\begin{aligned}
J_{222} \leq & C\|b\|_{C B M O^{p_{2}}\left(\mathbb{R}^{n}\right)}^{p_{2}} \sum_{k=-\infty}^{+\infty} \int_{B_{k}} \\
& \cdot\left|\sum_{i=k}^{+\infty}(i-k) \int_{C_{i}} \frac{\Omega(x-y) f(y)}{|y|^{n-\alpha}} d y\right|^{p_{2}} d x \\
\leq & C \sum_{k=-\infty}^{+\infty} \int_{B_{k}} \mid \sum_{i=k}^{+\infty}(i-k)\|f\|_{L^{p_{1}}\left(B_{i}\right)}\left(\int_{C_{i}}|\Omega(x-y)|^{q} d y\right)^{1 / q} \\
& \left.\cdot\left(\int_{C_{i}}|y|^{-(n-\alpha) s} d y\right)^{1 / s}\right|^{p_{2}} d x \\
\leq & C \sum_{k=-\infty}^{+\infty}\left|\sum_{i=k}^{+\infty} 2^{(k-i) n / p}\|f\|_{L^{p}\left(B_{i}\right)}\right|^{p} \\
\leq & C \sum_{i=-\infty}^{+\infty}\|f\|_{L^{p_{1}}\left(B_{i}\right)}^{p_{2}} \sum_{k=-\infty}^{i}(i-k) 2^{(k-i) n / 2} \\
\leq & C\|f\|_{L^{p_{1}}\left(\mathbb{R}^{n}\right)^{p^{n}},}^{p^{2}}
\end{aligned}
$$

which is the desired result and (a) is obtained.

Next, we verify (b) inspired by [18]. Namely, we need to show that there is a constant $C(\Omega, \alpha, p, n)$ such that

$$
\Theta:=\Theta(b, r):=\frac{1}{\left|B_{r}\right|} \int_{B_{r}}\left|b(y)-b_{B_{r}}\right| d y \leq C \text { for any } r \in \mathbb{R}^{+} .
$$

For abbreviation, we assume that $\left\|\left[b, H_{\Omega, \alpha}\right]\right\|_{L^{p_{1} \rightarrow L^{p_{2}}}}=1$, $\left\|\left[b, H_{\Omega, \alpha}^{*}\right]\right\|_{L^{p_{1}} \rightarrow L^{p_{2}}}=1$, and $b_{B_{r}}=0$ since $\left[b-b_{B_{r}}, H_{\Omega, \alpha}\right]=[b$, $\left.H_{\Omega, \alpha}\right]$. Let $f(y)=\operatorname{sgn}(b(y)) \chi_{B_{r}}(y)$. It is easy to check that $\left(1 /\left|B_{r}\right|\right) \int_{\mathbb{R}^{n}} f(y) b(y) d y=\Theta$.

Applying (3) and (10), we deduce that for $A$ and $\gamma$ appearing in (10), there is a constant $C_{1}<1$ such that $\sigma(D)$ $>0$ for $D=\left\{x^{\prime} \in \mathbb{S}^{n-1}: \Omega\left(x^{\prime}\right) \geq\left((2 A) /\left(\left(\log \left(2 / C_{1}\right)\right)^{\gamma}\right)\right)\right\}$. For $x^{\prime} \in D$ and $y^{\prime} \in \mathbb{S}^{n-1}$ with $\left|x^{\prime}-y^{\prime}\right| \leq C_{1}$, we obtain from (10) that

$$
\begin{aligned}
\Omega\left(y^{\prime}\right) & =\Omega\left(x^{\prime}\right)-\left(\Omega\left(x^{\prime}\right)-\Omega\left(y^{\prime}\right)\right) \\
& \geq\left|\Omega\left(x^{\prime}\right)\right|-\left|\Omega\left(x^{\prime}\right)-\Omega\left(y^{\prime}\right)\right| \\
& \geq \frac{A}{\left(\log \left(2 / C_{1}\right)\right)^{\gamma}} .
\end{aligned}
$$

Writing $E=\left\{x \in \mathbb{R}^{n}:|x|>C_{2} r\right.$ and $\left.\& x^{\prime} \in D\right\}$ with $C_{2}=$ $3 C_{1}^{-1}+1>4$, we see at once that for $x \in E,\left|\left[b, H_{\Omega, \alpha}\right] f(x)\right| \geq$ $\left|H_{\Omega, \alpha}(b f)(x)\right|-|b(x)|\left|H_{\Omega, \alpha} f(x)\right|:=K_{1}(x)-K_{2}(x)$. We conclude from (16) that $\left|(x-y)^{\prime}-x^{\prime}\right| \leq 3|y| /|x| \leq C_{1}$ since $|y|$ $<r$ and $|x|>C_{2}|y|>4|y|$, and hence, $\Omega\left((x-y)^{\prime}\right) \geq A$ / $\left(\log \left(2 / C_{1}\right)\right)^{\gamma}$, and finally, that 


$$
\begin{aligned}
K_{1}(x) & \geq\left|\frac{A}{\left(\log \left(2 / C_{1}\right)\right)^{\gamma}} \frac{1}{|x|^{n-\alpha}} \int_{|y|<|x|} b(y) f(y) d y\right| \\
& =\frac{A}{\left(\log \left(2 / C_{1}\right)\right)^{\gamma}|x|^{n-\alpha}} \int_{B_{r}} b(y) f(y) d y=\frac{C_{3} r^{n}}{|x|^{n-\alpha}} \Theta .
\end{aligned}
$$

Furthermore,

$$
\begin{aligned}
K_{2}(x) & \leq \frac{|b(x)|}{|x|^{n-\alpha}} \int_{B_{r}}|f(y)| \Omega\left((x-y)^{\prime}\right)-\Omega\left(x^{\prime}\right) \mid d y \\
& \leq \frac{C|b(x)|}{|x|^{n-\alpha}} \int_{B_{r}} \frac{|f(y)|}{(\log (|x| /|y|))^{\gamma}} d y \\
& \leq \frac{C|b(x)|}{|x|^{n-\alpha}} \int_{B_{r}} \frac{|f(y)|}{(\log (|x| / r))^{\gamma}} d y \\
& \leq \frac{C_{4} r^{n}|b(x)|}{|x|^{n-\alpha}(\log (|x| / r))^{\gamma}} .
\end{aligned}
$$

For abbreviation, we write

$$
\left\{\begin{array}{l}
F=\left\{x \in E:|b(x)|>\frac{C_{3} \Theta}{2 C_{4}}\left(\log \left(\frac{|x|}{r}\right)\right)^{\gamma},|x|<\Theta^{p_{2} / n} r\right\} ; \\
F_{1}=\{E \backslash F\} \cap\left\{|x|<\Theta^{p_{2} / n} r\right\} ; \\
F_{2}=\{E\} \cap\left\{C_{5}\left(|F|+\left(C_{2} r\right)^{n}\right)^{1 / n}<|x|<\Theta^{p_{2} / n} r\right\} .
\end{array}\right.
$$

This, along with the estimates for $K_{1}$ and $K_{2}$, one has

$$
\begin{aligned}
& \left\|\chi_{F}\right\|_{L^{p_{1}}\left(\mathbb{R}^{n}\right)}\|f\|_{L^{p^{\prime}}\left(\mathbb{R}^{n}\right)} \\
& \quad \geq\left\|\chi_{F}\right\|_{L^{p_{1}}\left(\mathbb{R}^{n}\right)}\left\|\left[b, H_{\Omega, \alpha}\right] f\right\|_{L^{p^{\prime}}\left(\mathbb{R}^{n}\right)} \\
& \quad \geq \int_{F_{1}}\left|\left[b, H_{\Omega, \alpha}\right] f(x)\right| d x \\
& \quad \geq \int_{F_{1}}\left|\frac{C_{3} r^{n} \Theta}{|x|^{n-\alpha}}-\frac{C_{3} r^{n} \Theta}{2|x|^{n-\alpha}}\right|^{p} d x \\
& \quad \geq\left(\frac{C_{3} r^{n} \Theta}{2}\right) \int_{C_{3}\left(|F|+\left(C_{2} r\right)^{n}\right)^{1 / n}}^{\Theta^{p_{2} / n} r} \rho^{\alpha-1} d \rho \int_{D} d \sigma\left(x^{\prime}\right) \\
& \quad \geq \sigma(D) \frac{\left(C_{3} r^{n} \Theta\right)}{\alpha}\left[\Theta^{\alpha p_{2} / n} r^{\alpha}-C_{5}^{\alpha}\left(|F|+\left(C_{2} r\right)^{n}\right)^{\alpha / n}\right] .
\end{aligned}
$$

Consequently,

$$
\begin{aligned}
\left(|F|+\left(C_{2} r\right)^{n}\right)_{\alpha / n} \geq & C_{5}^{-\alpha} \Theta^{\alpha p_{2} / n} r^{\alpha} \\
& -\frac{C_{5}^{-\alpha} \alpha}{C_{3} \sigma(D)} \frac{\left\|\chi_{F}\right\|_{L^{p_{1}}\left(\mathbb{R}^{n}\right)}\|f\|_{L^{p^{\prime}}\left(\mathbb{R}^{n}\right)}}{\Theta r^{n}} \\
\geq & C_{6} \Theta^{\alpha p_{2} / n} r^{\alpha} .
\end{aligned}
$$

This in turn implies that $C_{6} \Theta^{p_{2}} r^{n} \leq|F|+\left(C_{2} r\right)^{n}$. Thus, (b) is proved if $\Theta \leq\left(2 C_{6}^{-1} C_{2}^{n}\right)^{1 / p_{2}}$. If $\Theta>\left(2 C_{6}^{-1} C_{2}^{n}\right)^{1 / p_{2}}$, we see immediately that

$$
|F| \geq C_{6} \Theta^{p_{2}} r^{n}-C_{2}^{n} r^{n} \geq \frac{C_{6} \Theta^{p_{2}} r^{n}}{2} .
$$

According to $F \subset E, C_{2}>4,|y|>C_{2}|x|$, Lemma 2 and (37), we can obtain that for $x \in B_{r}$ and $y \in F$,

$$
|x-y| \approx|y|, \Omega\left((x-y)^{\prime}\right) \geq \frac{A}{\left(\log \left(2 / C_{1}\right)\right)^{\gamma}} .
$$

We continue to choose $f^{*}(y)=(\operatorname{sgn}(b(y))) \chi_{F}(y)$ for $x \in B_{r}$ and get

$$
\begin{aligned}
\left|\left[b, H_{\Omega, \alpha}^{*}\right] f^{*}(x)\right| \geq & \int_{F} \frac{|\Omega(x-y) b(y)|}{|y|^{n-\alpha}} d y \\
& -|b(x)| \int_{F} \frac{\left|\Omega(x-y) f^{*}(y)\right|}{|y|^{n-\alpha}} d y \\
:= & L_{1}(x)+L_{2}(x) .
\end{aligned}
$$

It is easy to check that

$$
\begin{aligned}
L_{2}(x) & \leq C_{7}|b(x)| \int_{C_{2} r}^{\Theta^{\rho_{2} / n} r} \rho^{\alpha-1} d \rho \int_{\mathbb{S}^{n-1}} d \sigma\left(y^{\prime}\right) \\
& \leq C_{8}|b(x)|\left(\Theta^{\alpha p_{2} / n} r^{\alpha}-C_{2}^{\alpha} r^{\alpha}\right) .
\end{aligned}
$$

To deal with the term $L_{1}(x)$, we first obtain from (44) that

$$
L_{1}(x) \geq \frac{A C_{3} \Theta}{2 C_{4}\left(\log 1 / C_{1}\right)^{\gamma}} \int_{F} \frac{(\log (|y| / r))^{\gamma}}{|y|^{n-\alpha}} d y .
$$

Then, the estimate for $L_{1}(x)$ consists of two cases.

Case 1. $\gamma \geq n$. Since $4 r<C_{2} r<|y|<\Theta^{p_{2} / n} r$ for $y \in F$, we conclude from (47) and $|F| \geq\left(C_{6} \Theta^{p_{2}} r^{n}\right) / 2$ that

$$
\begin{aligned}
L_{1}(x) & \geq \frac{C_{9} \Theta}{r^{n}} \int_{F}\left(\frac{\log (|y| / r)}{|y| / r}\right)^{n} \frac{(\log (|y| / r))^{\gamma-n}}{|y|^{\alpha}} d y \\
& \geq \frac{C_{9} \Theta^{1+\left(\alpha p_{2} / n\right)}}{r^{n-\alpha}}\left(\frac{\log \left(p_{2} / n\right)}{\Theta^{p_{2} / n}}\right)^{n}\left(\log C_{2}\right)^{\gamma-n}|F| \\
& \geq C_{10} \Theta^{1+\alpha p_{2} / n}(\log \Theta)^{n} r^{\alpha} .
\end{aligned}
$$

Case 2. $1<\gamma<n$. In this case,

$$
\begin{aligned}
L_{1}(x) & \geq \frac{C_{9} \Theta^{1+\left(\alpha p_{2} / n\right)}}{r^{n-\alpha}} \int_{F} \frac{(\log (|y| r))^{\gamma}}{(|y| / r)^{n}} d y \\
& \geq C_{11} \Theta^{1+\alpha p_{2} / n}(\log \Theta)^{\gamma} r^{\alpha} .
\end{aligned}
$$

This in turn reveals that

$$
\begin{aligned}
\left|\left[b, H_{\Omega, \alpha}^{*}\right] f^{*}(x)\right| \geq & C_{11} \Theta^{1+\alpha p_{2} / n}(\log \Theta)^{\gamma} r^{\alpha} \\
& -C_{9}|b(x)| r^{\alpha} \Theta^{\alpha p_{2} / n}, \quad \forall x \in B_{r} .
\end{aligned}
$$


Having disposed of the above estimates, we can now obtain

$$
\begin{aligned}
C_{12} r^{n+\alpha} \Theta^{1+\alpha p_{2} / n} \geq & \left(\int_{|x|<\Theta^{p_{2} / n} r} d x\right)^{1 / p_{1}} r^{n / p_{2}^{\prime}} \geq r^{n / p_{2}^{\prime}}\left\|f^{*}\right\|_{L^{p_{1}}\left(\mathbb{R}^{n}\right)} \\
\geq & \left\|\chi_{B_{r}}\right\|_{L^{p_{2}^{\prime}\left(\mathbb{R}^{n}\right)}}\left\|\left[b, H_{\Omega, \alpha}^{*}\right] f^{*}\right\|_{L^{p_{2}\left(\mathbb{R}^{n}\right)}} \\
\geq & \int_{B_{r}}\left|\left[b, H_{\Omega, \alpha}^{*}\right] f^{*}\right| d x \\
\geq & C_{11} \Theta(\log \Theta)^{\gamma} r^{\alpha} \int_{B_{r}} d x \\
& -C_{9} \Theta^{\alpha p_{2} / n} r^{\alpha} \int_{B_{r}}|b(x)| d x \\
\geq & C_{11} \Theta^{1+\alpha p_{2} / n} r^{n+\alpha}(\log \Theta)^{\gamma}-C_{9} \Theta^{1+\alpha p_{2} / n} r^{n+\alpha} .
\end{aligned}
$$

Namely, $C_{11}(\log \Theta)^{\gamma}-C_{9} \leq C_{12}$. This in turn shows that there is a constant $C:=C(\Omega, \alpha, n, p, \gamma)$ such that $\Theta \leq C$, whence reaching the desired fact.

\section{Data Availability}

The data used to support the findings of this study are included within the article.

\section{Conflicts of Interest}

The author declares that there are no conflicts of interest.

\section{Acknowledgments}

The authors would like to thank the NSF of China (Grant no. 11771195), the NSF of Shandong Province (Grant nos. ZR2019YQ04, 2020KJI002, and 2019KJI003) and the Key Laboratory of Complex Systems and Intelligent Computing in University of Shandong (Linyi University) for the financial support. We are grateful to the referee for his/her comments and suggestions to improve this paper.

\section{References}

[1] Z. W. Fu, S. Z. Lu, and F. Y. Zhao, "Commutators of $n$-dimensional rough Hardy operators," Science China. Mathematics, vol. 54, no. 1, pp. 95-104, 2011.

[2] Z. W. Fu, Z. G. Liu, S. Z. Lu, and H. B. Wang, "Characterization for commutators of $n$-dimensional fractional Hardy operators," Science in China Series A: Mathematics, vol. 50, no. 10, pp. 1418-1426, 2007.

[3] L. Shanzhen and Y. Dachun, "The central BMO spaces and Littlewood-Paley operators," Approximation Theory and its Applications, vol. 11, no. 3, pp. 72-94, 1995.

[4] Y. Komori, "Notes on commutators on Herz-type spaces," Archiv der Mathematik, vol. 81, no. 3, pp. 318-326, 2003.

[5] M. Bramanti and M. C. Cerutti, " $W_{p}^{1,2}$ solvability for the Cauchy-Dirichlet problem for parabolic equations with VMO coefficients," Communications in Partial Differential Equations, vol. 18, no. 9-10, pp. 1735-1763, 2007.
[6] F. Chiarenza, M. Frasca, and P. Longo, " $W^{2, p}$-solvability of the Dirichlet problem for nondivergence elliptic equations with VMO coefficients," Transactions of the American Mathematical Society, vol. 336, no. 2, pp. 841-853, 1993.

[7] T. Gadjiev, S. Galandarova, and V. Guliyev, "Regularity in generalized Morrey spaces of solutions to higher order nondivergence elliptic equations with VMO coefficients," Electronic Journal of Qualitative Theory of Differential Equations, vol. 55, no. 55, pp. 1-17, 2019.

[8] A. Scapellato, "Regularity of solutions to elliptic equations on Herz spaces with variable exponents," Boundary Value Problems, vol. 2019, no. 1, 2019.

[9] A. Scapellato, "Homogeneous Herz spaces with variable exponents and regularity results," Electronic Journal of Qualitative Theory of Differential Equations, no. 82, pp. 1-11, 2018.

[10] Y. Sun, C. Wang, and Z. Zhang, "A Beale-Kato-Majda blowup criterion for the 3-D compressible Navier-Stokes equations," Journal de Mathématiques Pures et Appliquées, vol. 95, no. 1, pp. 36-47, 2011.

[11] F. Zhao and S. Lu, "A characterization of $\lambda$-central BMO space," Frontiers of Mathematics in China, vol. 8, no. 1, pp. 229-238, 2013.

[12] R. R. Coifman, R. Rochberg, and G. Weiss, "Factorization theorems for hardy spaces in several variables," The Annals of Mathematics, vol. 103, no. 3, pp. 611-635, 1976.

[13] S. Janson, "Mean oscillation and commutators of singular integral operators," Arkiv för Matematik, vol. 16, no. 1-2, pp. 263270, 1978

[14] M. Paluszynski, "Characterization of the Besov spaces via the commutator operator of Coifman, Rochberg and Weiss," Indiana University Mathematics Journal, vol. 44, no. 1, pp. 1-18, 1995.

[15] S. Shi and S. Lu, "Characterization of the central Campanato space via the commutator operator of hardy type," Journal of Mathematical Analysis and Applications, vol. 429, no. 2, pp. 713-732, 2015.

[16] Y. Chen and Y. Ding, "A charaterization of commutators for parabolic singular integrals," Mathematica Scandinavica, vol. 108, no. 1, pp. 5-25, 2011.

[17] D. S. Kurtz and R. L. Wheeden, "Results on weighted norm inequalities for multipliers," Transactions of the American Mathematical Society, vol. 255, pp. 343-362, 1979.

[18] Y. P. Chen and Y. Ding, "Compactness of the commutators of parabolic singular integrals," Science China Mathematics, vol. 53 , no. 10 , pp. $2633-2648,2010$.

[19] A. P. Calderón, M. Weiss, and A. Zygmund, "On the existence of singular integrals," Singular Integrals, vol. 10, pp. 56-73, 1967. 